\title{
CCN5 overexpression inhibits profibrotic phenotypes via the PI3K/Akt signaling pathway in lung fibroblasts isolated from patients with idiopathic pulmonary fibrosis and in an in vivo model of lung fibrosis
}

\author{
LIN ZHANG, YINGNA LI, CHUNLIAN LIANG and WEILIN YANG \\ Department of Geriatrics, The Second Affiliated Hospital, Medical School of \\ Xi'an Jiaotong University,Xi'an, Shaanxi 710004, P.R. China
}

Received September 10, 2013; Accepted November 21, 2013

DOI: 10.3892/ijmm.2013.1565

\begin{abstract}
Idiopathic pulmonary fibrosis (IPF) is a chronic and progressive interstitial lung disease with unknown etiology and undefined treatment modality. Fibroblasts are regarded as the major cell type that mediates the onset and progression of lung fibrosis by secreting large amounts of extracellular matrix (ECM) proteins, such as connective tissue growth factor (CTGF/CCN2). Current knowledge confers a crucial role of $\mathrm{CCN} 2$ in lung fibrosis. CCN5, another member of the $\mathrm{CCN}$ family, has been suggested to play an inhibitory role in some fibrotic diseases, such as cardiac fibrosis. However, the role of CCN5 in the process of IPF remains unknown. In the present study, using western blot analysis, we demonstrate that CCN2 is highly expressed in fibroblasts derived from IPF tissue, but is only slightly expressed in normal human lung fibroblasts. However, CCN5 was weakly expressed in all the above cells. qRT-PCR revealed that transforming growth factor (TGF)- $\beta 1$ stimulation increased CCN2 expression in the IPF-derived cultures of primary human lung fibroblasts (PIFs) in a time- and concentration-dependent manner, but only slightly affected the expression of CCN5. The overexpression of CCN5 induced by the transfection of PIFs with recombinant plasmid did not affect cell viability, proliferation and apoptosis; however, it significantly suppressed the expression of CCN2, $\alpha$-smooth muscle actin ( $\alpha$-SMA) and collagen type I. The TGF- $\beta 1$-induced upregulation of the phosphorylation of Akt was reversed by CCN5 overexpression. Our results also demonstrated that adenovirus-mediated CCN5 overexpression
\end{abstract}

Correspondence to: Dr Weilin Yang, Department of Geriatrics, The Second Affiliated Hospital, Medical School of Xi'an Jiaotong University, 157 Xiwu Road, Xi'an, Shaanxi 710004, P.R. China E-mail: weilinyangxian@163.com

Key words: idiopathic pulmonary fibrosis, fibroblast, CCN5, CCN2, transforming growth factor- $\beta$ in a mouse model of bleomycin-induced IPF significantly decreased the hydroxyproline content in the lungs, as well as TGF- $\beta 1$ expression in bronchoalveolar lavage fluid. Taken together, our data demonstrate that CCN5 exerts an inhibitory effect on the fibrotic phenotypes of pulmonary fibroblasts in vitro and in vivo, and as such may be a promising target for the treatment of IPF.

\section{Introduction}

Idiopathic pulmonary fibrosis (IPF) is the most common form of interstitial lung disease, and generally has a poor prognosis, with an average survival of 3-5 years following diagnosis (1). The etiology underlying IPF is incompletely understood and the therapeutic use of drugs for IPF treatment remains poorly elucidated and controversial. Although multiple processes and cells may be involved in the pathogenesis of IPF, certain studies have emphasized the role of fibroblasts in IPF (2). In some unknown conditions, such as chronic inflammation, resident lung fibroblasts are activated and are transformed into myofibroblasts, which are more contractile, proliferative and secretory active (3). These cells are also characterized by an increased expression of $\alpha$-smooth muscle actin ( $\alpha$-SMA) and extracellular matrix (ECM) proteins, such as fibronectin, collagen and connective tissue growth factor (CTGF)/ CCN2) $(4,5)$. Transforming growth factor (TGF)- $\beta$ is regarded as the most potent mediator stimulating matrix production and fibroblast-to-myofibroblast differentiation and is considered to play a critical role in the pathogenesis of IPF $(6,7)$.

CCN2, which is also known as CTGF, belongs to the connective tissue growth factor/cysteine-rich 61/nephroblastoma overexpressed (CCN) family, which is composed of 6 members, including CCN1 [cysteine-rich, angiogenic inducer, 61 (CYR61)], CCN2 (CTGF), CCN3 [nephroblastoma overexpressed (NOV)], CCN4 [Wnt-induced secreted protein (WISP)-1], CCN5 (WISP-2) and CCN6 (WISP-3) $(8,9)$. These members of the $\mathrm{CCN}$ family were grouped on the basis of similar structural analogies, which encompass 4 conserved structural modules: an insulin-like growth factor binding protein (module I), a von Willebrand factor (module II), a 
thrombospondin-homology domain (module III) and a cysteine knot (CT) (module IV), apart from CCN5 (8). CCN2 has been verified to play an important role in a variety of cellular processes, including neovascularization, neuronal scarring, embryogenesis, transdifferentiation and wound healing (10). As an essential downstream mediator of the physiological effects of TGF- $\beta$ signaling, CCN2 is also deemed as a component of the profibrotic matrix $(2,5,11)$. The profibrotic properties of CCN2 in IPF, as well as other fibrotic diseases have been well established (12). Transgenic mice overexpressing CCN2 in fibroblasts have been shown to develop fibrosis in multiple organs (13), whereas mice bearing a fibroblast/smooth muscle cell-specific deletion of CCN2 are resistant to bleomycininduced skin fibrosis (14). Previous studies have demonstrated that CCN2 expression is increased in fibroblasts isolated from patients with IPF (15); the overexpression of CCN2 is crucial to inducing a profibrotic environment in mouse lungs (16). Recently, Kono et al (5) demonstrated that plasma CCN2 levels are significantly higher in patients with IPF compared with healthy volunteers, suggesting that $\mathrm{CCN} 2$ is a potential biomarker for IPF.

CCN5, another member of the CCN family, also known as WISP-2, is a $29-\mathrm{kDa}$ matrix protein encoded by the WISP-2/ CCN5 gene located on chromosome 20q12-q13.1 (17). However, CCN5 contains only 3 structural modules and lacks the CT domain (18). These data suggest that CCN5 acts as a dominantnegative protein to suppress CCN2-mediated fibrogenesis (19). It was recently demonstrated that in isolated cardiomyocytes, the overexpression of $\mathrm{CCN} 2$ induced hypertrophic growth, whereas the overexpression of CCN5 inhibited CCN2-induced hypertrophic responses (20). TGF- $\beta$-Smad signaling was elevated in CCN2 transgenic mice, but was inhibited in CCN5 transgenic mice (20). These data indicate the opposing roles of CCN2 and CCN5 in cardiac hypertrophy and fibrosis. Another study demonstrated that CCN5 acts as a transcriptional repressor and represses the expression of genes associated with key components of the TGF- $\beta$ signaling pathway (21), which is the dominant pathway in fibrosis. However, the role of CCN5 in IPF remains largely unknown. Based on the data mentioned above, we hypothesized that the overexpression of CCN5 in lungs would prevent the progression of IPF.

In the present study, we aimed to investigate the role of CCN5 in IPF. We demonstrate that the overexpression of CCN5 in lung fibroblasts suppresses the upregulation in the expression of $\alpha$-SMA and collagen induced by CCN2. Furthermore, CCN5 overexpression in lung fibroblasts decreased the expression of CCN2. The PI3K/Akt signaling pathway may be involved in the anti-fibrotic effects of CCN5. By constructing an adenovirus-mediated CCN5 expression vector, we also demonstrate that CCN5 alleviates lung fibrosis in a mouse model of bleomycin-induced IPF.

\section{Materials and methods}

Antibodies. Mouse anti-human CCN2 monoclonal antibody (Ab), mouse anti-human CCN5 monoclonal Ab, mouse anti-human collagen I monoclonal $\mathrm{Ab}$, mouse anti-human $\alpha$-SMA monoclonal Ab, mouse anti-phospho human Akt1 (phospho S473) monoclonal Ab, mouse anti-human Akt1 monoclonal Ab, rabbit anti-phospho human JNK1 (phospho
T183+Y185) polyclonal Ab, rabbit anti-human JNK1 polyclonal $\mathrm{Ab}$, rabbit anti-human Smad2 polyclonal $\mathrm{Ab}$, mouse anti-human $\alpha$-tubulin monoclonal $\mathrm{Ab}$, rabbit anti- $\beta$-actin polyclonal $\mathrm{Ab}$ and horseradish peroxidase (HRP)-conjugated rabbit anti-mouse immunoglobulin $\mathrm{G}(\mathrm{IgG})$ were all purchased from Abcam (Cambridge, MA, USA). Rabbit anti-phospho human Smad2 (phospho S465/S467) polyclonal Ab was purchased from Cell Signaling Technology Inc. (Beverly, MA, USA).

Isolation and culture of human pulmonary fibroblasts. Human lung fibroblasts were isolated from lung biopsies following enzymatic disaggregation by collagenase and protease as previously described (22) to obtain cell cultures of primary fibroblasts. Among these, human lung fibroblasts were isolated from a patient with IPF (PIFs) who had never been treated with steroids or immunosuppressive drugs at the time of the biopsy. Primary human normal lung fibroblasts (PNFs) isolated from patients undergoing thoracic surgery for non-fibrotic lung diseases were used as the control fibroblasts. This study was approved by the Ethics Committee of Xi'an Jiaotong University, Xi'an, China, and written informed consent was obtained on all patients prior to the procedure being performed. In addition, 2 adult human IPF-derived lung fibroblast cell lines, LL-97A and LL-29, as well as 1 normal human lung fibroblast cell line, LL-24, were obtained from the American Type Cell Culture Collection (ATCC; Manassas, VA, USA).

Fibroblast cell cultures and cell lines were cultured in Dulbecco's modified Eagle's medium (DMEM) containing $10 \%$ heat-inactivated fetal calf serum, $100 \mathrm{U} / \mathrm{ml}$ penicillin, $100 \mu \mathrm{g} / \mathrm{ml}$ streptomycin and $2 \mathrm{mM} \mathrm{L}$-glutamine at $37^{\circ} \mathrm{C}$ in a $5 \% \mathrm{CO}_{2}$ environment. The culture medium was changed every 2-3 days until the cells reached confluence. Cultures were used in this study after $4-8$ passages. For TGF- $\beta$ stimulation, fibroblast cell cultures and cell lines kept in serum-free medium were also treated with various concentrations of TGF- $\beta 1$ (Sigma-Aldrich, St. Louis, MO, USA) ranging from 0.5 to $8 \mathrm{ng} / \mathrm{ml}$ for 6-72 h.

For the signaling pathway assay, the PI3K inhibitor, LY294002 (Sigma-Aldich), was added to the cultures $1 \mathrm{~h}$ prior to TGF- $\beta 1$ stimulation. After $24 \mathrm{~h}$, the cells were harvested for further assays.

$R N A$ extraction and quantitative reverse transcription PCR ( $q R T-P C R)$. Total RNA from human lung fibroblasts was extracted using TRIzol reagent (Invitrogen Life Technologies, Carlsbad, CA, USA), based on the manufacturer's instructions. cDNA was synthesized by the reverse transcription of total RNA, using the PrimeScript RT reagent kit (Takara Bio, Inc., Dalian, China) using oligo(dT) primers, following the manufacturer's instructions. (qRT-PCR) reactions were performed on a Bio-Rad (Hercules, CA, USA) iQ5 real-time thermal cycler using the SYBR Premix Ex Taq ${ }^{\mathrm{TM}}$ II kit (Takara Bio, Inc.). The qRT-PCR reaction system and cycling parameters were based on the Thermal Cycler Dice Real Time System. Twenty nanograms of cDNA and $0.4 \mu \mathrm{M}$ primers were included in the $25 \mu \mathrm{l}$ of amplification mixture. The PCR primers used were as follows: CCN2, 5'-ACCCCTGCGACCCGCACAAG-3' (forward) and 5'-TTCACTTGCCACAAGCTGTC-3' (reverse); CCN5, 5'-CCTCTCAAAGGTGCGTACCCA-3' (forward) and 5'-CACGGACCATCTTCCATCAGC-3' (reverse); $\beta$-actin, 
5'-CAACTTGATGTATGAAGGCTTTGGT-3' (forward) and 5'-ACTTTTATTGGTCTCAAGTCAGTGTACAG-3' (revers e). These primers were all synthesized by Sangon Biotech Co., Ltd. (Shanghai, China). The PCR procedure was as follows: polymerase activation for $30 \mathrm{sec}$ at $95^{\circ} \mathrm{C}, 40$ cycles of amplification each consisting of $95^{\circ} \mathrm{C}$ for $5 \mathrm{sec}, 60^{\circ} \mathrm{C}$ for $20 \mathrm{sec}$, and 1 cycle of dissociation consisting of $95^{\circ} \mathrm{C}$ for $15 \mathrm{sec}, 60^{\circ} \mathrm{C}$ for $30 \mathrm{sec}$ and $95^{\circ} \mathrm{C}$ for $15 \mathrm{sec}$. All reactions were performed in triplicate, and the results were represented as relative mRNA expression data calculated according to the $2^{-\Delta \Delta C T}$ method, as previously described (23).

Western blot analysis. The proteins were extracted from fibroblast cells using RIPA lysis buffer (Beyotime, Nantong, China). The protein concentration in the lysates was determined using a BCA protein assay kit (Beyotime). For western blot analysis, proteins were separated on an SDS-polyacrylamide gel, transferred onto nitrocellulose membranes (Amersham Pharmacia Biotech, Piscataway, NJ,. USA) in a semi-dry transblot apparatus by transfer buffer, blocked with $10 \%$ defatted milk in PBS at $4^{\circ} \mathrm{C}$ overnight, and then incubated with the primary antibodies for $60 \mathrm{~min}$. After washing with TBST buffer $(0.05 \mathrm{~mol} / 1$ Tris, $0.15 \mathrm{~mol} / 1 \mathrm{NaCl}$ and $0.05 \%$ Tween-20), the membranes were incubated for $1 \mathrm{~h}$ at room temperature with HRP-conjugated secondary Ab. For detection, the ECL reagent (Boehringer Mannheim, Mannheim, Germany) was used. All experiments were performed in triplicate, and results were normalized to $\beta$-actin.

Plasmid construction and cell transfection. Plasmid construction and cell transfection were performed as previously described (24). Briefly, total cDNA was obtained as described above. The open reading frame of the CCN5 cDNA was then cloned and inserted in the pCEP4-Flag vector (Invitrogen Life Technologies) to generate the pCEP4-Flag-CCN5 recombinant plasmid. For cell transfection, cells were cultured to $60 \%$ confluence and transfection were performed using the FuGENE HD Transfection Reagent (Roche, Indianapolis, IN, USA) as suggested by the manufacturer.

Cell viability assay. Cell viability assays were performed using a CellTiter-Blue cell viability assay kit (Promega Corp., Madison, WI, USA) according to the manufacturer's instructions.

$\left[{ }^{3} H\right]$-thymidine incorporation assay $\left(\left[{ }^{3} H\right]-T d R\right)$. Cell proliferation was measured using the $\left[{ }^{3} \mathrm{H}\right]-\mathrm{TdR}$ assay as previously described (7). Cells were cultured in triplicate at $4 \times 10^{4}$ cells/ well (24-well plate) in DMEM medium containing $10 \%$ fetal calf serum and cultured for $24 \mathrm{~h}$. Cells were rinsed, serumstarved in DMEM for $2 \mathrm{~h}$, and treated in serum-free DMEM with the indicated treatments. $\left[{ }^{3} \mathrm{H}\right]$-thymidine (Sigma-Aldich) at $2 \mu \mathrm{Ci} / \mathrm{ml}$ was added followed by incubation for $6 \mathrm{~h}$. Then cells were washed twice with ice-cold normal saline followed by the addition of $10 \%$ trichloroacetic acid for $30 \mathrm{~min}$ at $4^{\circ} \mathrm{C}$. The radioactivity was determined using a liquid scintillation counter (Beckman Coulter, Fullerton, CA, USA).

Apoptosis assay. Cell apoptosis was evaluated by measuring the activity of caspase-3/7. Caspase-3/7 activity was deter- mined using the Caspase-Glo 3/7 assay kit (Promega Corp.) following the manufacturer's instructions. Briefly, the fibroblasts treated with the various treatments were plated on 96-well plates at a volume of $100 \mu \mathrm{l}$. Caspase-Glo 3/7 reagent was added to each well in a 1:1 ratio and incubated with gentle shaking for $30 \mathrm{~min}$ at room temperature before measuring luminescence using a microplate reader (Corona Electric Co., Ltd., Katsuta, Japan).

Adenovirus construction and transfection. All recombinant adenoviruses were constructed as described in a previous study (19). Briefly, the full-length CCN5 cDNA was cloned into the adenoviral shuttle plasmid, pAdTrack-CMV, which contained the gene encoding green fluorescent protein (GFP). The recombinant shuttle plasmids, pAdTrack-CMV and pAdEasy-1, were then homologously recombined in the Escherichia coli strain, BJ5183. The obtained recombinant plasmids were transfected into HEK293 cells to generate the recombinant adenovirus, Ad-CCN5. This virus was amplified and purified, titered by a p24 ELISA kit (Cell Biolabs, Inc., San Diego, CA, USA) and stored at $-80^{\circ} \mathrm{C}$. Ad-GFP was also generated and used as the negative control.

Establishment of a mouse model of bleomycin-induced IPF and treatment of mice. C57BL/6 mice (8 weeks old) were provided by the Laboratory Animal Center of Xi'an Jiaotong University. All mice were housed in specific pathogen-free conditions with free access to water. All the experiments were carried out in adherence with a protocol approved by the Institutional Animal Care and Use Committee of Xi'an Jiaotong University. The mouse model of IPF was established as previously described $(1,25)$. Briefly, 8-week-old male C57BL/6 mice were lightly anesthetized with sodium pentobarbital (dose, 50 mg/kg; Dainippon Pharmaceutical, Co. Ltd., Osaka, Japan). They were then treated with either normal saline or bleomycin sulfate $(2 \mathrm{mg} / \mathrm{kg}$ of body weight; SigmaAldrich) dissolved in saline by an intratracheal injection in a total volume of $20 \mu \mathrm{l}$. To evaluate the ability of the adenoviral vector-mediated overexpression of CCN5 to attenuate the fibrotic changes induced by bleomycin, the mice were lightly anesthetized with inhaled diethyl ether (Wako Pure Chemical Industries, Ltd., Osaka, Japan) and intranasally administered $200 \mu \mathrm{l}$ Ad-CCN5 virus $\left[1 \times 10^{10}\right.$ plaque-forming units (pfu) of virus] 2 days pior to treatment with bleomycin. The mice in the normal control group were administered normal saline and the mice in the negative control group were administered Ad-GFP virus 2 days prior to treatment with bleomycin. On the 14th day, the mice were sacrificed and the lungs were collected for hydroxyproline assay.

Hydroxyproline assay. To estimate the total amount of collagen in the lungs, we measured the amount of hydroxyproline in lung tissue according to a previously described protocol $(26,27)$. Lung tissue samples were frozen in liquid nitrogen and homogenized in $2 \mathrm{ml}$ of $0.5 \mathrm{~mol} / 1$ glacial acetic acid. One milliliter of the homogenate was hydrolyzed in $2 \mathrm{ml}$ of $6 \mathrm{~mol} / \mathrm{l} \mathrm{HCl}$ for $16 \mathrm{~h}$ at $110^{\circ} \mathrm{C}$. Hydroxyproline content was determined by a colorimetric assay using a spectrophotometer at a wavelength of $550 \mathrm{~nm}$, and absolute values were determined from a standard curve. 
ELISA. The detection of TGF- $\beta 1$ in bronchoalveolar lavage fluid (BALF) was performed as previously described (28). For BALF collection, a 23-gauge cannula was inserted into the trachea on day 14 and the lungs were lavaged 5 times with $0.8 \mathrm{ml}$ of PBS. The collected fluid was centrifuged at $1,000 \mathrm{x} \mathrm{g}$ for $10 \mathrm{~min}$. The collected BALF was divided into aliquots and stored at $-80^{\circ} \mathrm{C}$. The levels of TGF- $\beta 1$ in BALF were measured with an ELISA kit (R\&D Systems, Minneapolis, MN, USA) using a standardized procedure.

Statistical analysis. Data are expressed as the means \pm SEM from a minimum of 3 experiments. The Student's t-test was used to determine the significance of differences in multiple comparisons. A value of $\mathrm{P}<0.05$ was considered to indicate a statistically significant difference.

\section{Results}

TGF- $\beta 1$ stimulation upregulates the expression of CCN2 but not CCN5 in human lung fibroblasts. In light of the profibrotic function of $\mathrm{CCN} 2$ in pulmonary fibrosis and the putative role of CCN5 in the inhibition of fibrosis, we sought to measure the expression levels of CCN2 and CCN5 by western blot analysis in human pulmonary fibroblast cells. As expected, CCN2 was highly expressed in the PIFs and the human IPF lung fibroblastic cell lines, LL-97A and LL-29, but weakly expressed in the PNFs and the normal human lung fibroblastic cell line, LL-24 (Fig. 1A). However, CCN5 expression was low in all the above cells (Fig. 1A). As it has been reported that TGF- $\beta$ is a potent inducer of CCN2 expression (11), as well as various types of fibrosis, we wished to determine whether CCN5 expression is affected by TGF- $\beta 1$ stimulation in PIFs. As revealed by qRT-PCR, the transcription of CCN2 mRNA was markedly upregulated, accompanied by the increased TGF- $\beta 1$ concentrations; however, CCN5 expression was only slightly altered (Fig. 1B). After $12 \mathrm{~h}$ of stimulation with $2 \mathrm{ng} / \mathrm{ml}$ TGF- $\beta 1$, the transcription of CCN2 mRNA was significantly increased. However, the transcription of CCN5 mRNA hardly changed as time progressed (Fig. 1C). These data indicated that TGF- $\beta 1$ stimulation significantly increased CCN2 expression in a concentration- and time-dependent manner. To exclude a cell line-specific difference, CCN2 and CCN5 expression patterns in response to TGF- $\beta 1$ were also determined in the LL-97A, LL-29 cells, as well as in PNFs. As expected, TGF- $\beta 1$-stimulation markedly elevated CCN2 expression but had little effect on CCN5 expression in all cell lines examined (Fig. 1D and E).

CCN5 overexpression does not affect the viability, proliferation or apoptosis of pulmonary fibroblasts. As we only detected a weak expression of CCN5 and its minimal alteration in response to TGF- $\beta 1$, we explored the role of CCN5 by inducing the overexpression of CCN5 in PIFs. qRT-PCR and western blot analysis demonstrated that the mRNA and protein levels of CCN5, respectively were both significantly elevated by transfection with the pCEP4-Flag-CCN5 vector compared with the control group (Fig. 2A and B). Furthermore, the mRNA level of CCN2 was markedly downregulated by CCN5 overexpression (Fig. 2A). The protein expression of CCN2 was also inhibited, although no significant difference was observed (Fig. 2B). We then demonstrated that transfection with pCEP4-Flag-CCN5 did not affect cell viability by a colorimetric method based on the tetrazolium salt, WST-8 (Fig. 2C). Using a $\left[{ }^{3} \mathrm{H}\right]$-thymidine incorporation assay, we also revealed that the proliferation of pulmonary fibroblasts was not significantly affected following transfection with pCEP4-Flag-CCN5 compared with the control group, even after TGF- $\beta 1$-stimulation (Fig. 2D). Caspase-3/7 activity in the pulmonary fibroblasts was only slightly altered by transfection with pCEP4-Flag-CCN5 compared with the control group, regardless of TGF- $\beta 1$-stimulation (Fig. 2E). The overexpression of CCN5 in the LL-97A and LL-29 cells also suppressed the expression of CCN2, whereas it did not affect cell viability, proliferation or apoptosis (data not shown).

CCN5 overexpression inhibits the profibrotic phenotype of lung fibroblasts. Fibroblast-to-myofibroblast differentiation is a critical process in the pathogenesis of a number of fibrotic diseases, including IPF. To determine whether CCN5 expression affects this process, the PIFs were transfected with pCEP4-Flag-CCN5 and then their differentiation was induced with $2 \mathrm{ng} / \mathrm{ml}$ TGF- $\beta 1$ for $24 \mathrm{~h}$. Western blot analysis demonstrated that the expression of $\alpha$-SMA, a biomarker of myofibroblast differentiation, was markedly downregulated by CCN5 overexpression (Fig. 3A). This indicated that CCN5 hindered the TGF- $\beta 1$-induced myofibroblast differentiation.

ECM accumulation is a prominent characteristic of the process of fibrosis. Therefore, the PIFs transfected with pCEP4Flag-CCN5 plasmid were stimulated with $2 \mathrm{ng} / \mathrm{ml}$ TGF- $\beta 1$ for $24 \mathrm{~h}$, and the alteration in the expression of collagen type I was examined by western blot analysis. Compared with the control group, collagen type I expression was markedly reduced by CCN5 overexpression (Fig. 3B). Taken together, these data suggested that the profibrotic phenotype of lung fibroblasts was alleviated by CCN5 overexpression.

The PI3K/Akt pathway is involved in the anti-fibrotic role of $C C N 5$. It has been previously suggested that the Smads, and the PI3K/Akt and JNK pathway are involved in TGF- $\beta 1$ induced fibrosis (29). Thus, in this study, we investigated whether CCN5 exerts its anti-fibrotic role by blocking these signaling pathways. TGF- $\beta 1$ stimulation markedly increased the phosphorylation of Smad2, JNK and Akt1 (Fig. 4). Of note, transfection with pCEP4-Flag-CCN5 reduced the phosphorylation of Smad 2 and JNK induced by TGF- $\beta 1$ stimulation, although not significantly (Fig. 4). However, the upregulated phosphorylation of Akt1 induced by TGF- $\beta 1$ was markedly attenuated by transfection with pCEP4-Flag-CCN5 (Fig. 4A and D). We also demonstrated that the effects of CCN5 overexpression were similar to those of the PI3K inhibitor, LY294002 (Fig. 4). These data indicated that, to a greater or lesser extent, the PI3K/Akt pathway is involved in the inhibitory effects of CCN5 on lung fibrosis.

Overexpression of CCN5 alleviates bleomycin-induced lung fibrosis in a mouse model. To determine the inhibitory effects of CCN5 overexpression on lung fibrosis in vivo, a mouse model of bleomycin-induced lung fibrosis was established. An adenoviral vector expressing human CCN5 was also generated and used to infect the mice 2 days prior to treatment with 
A

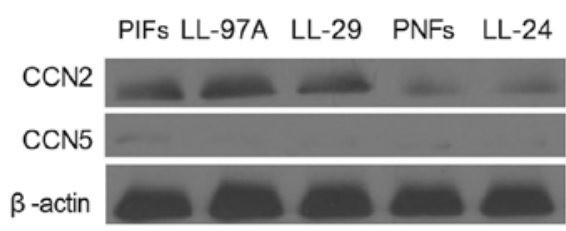

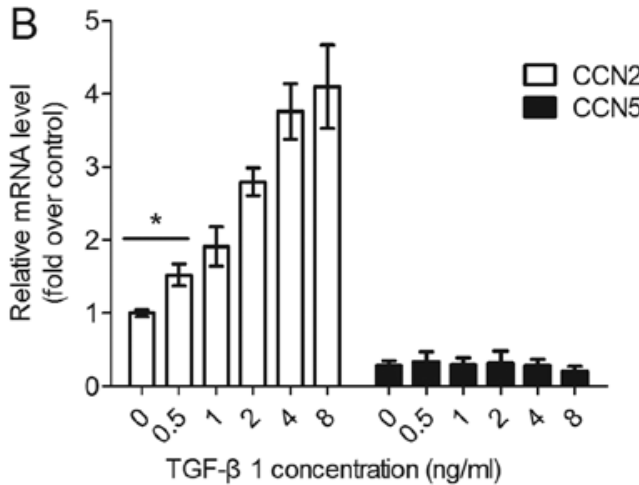

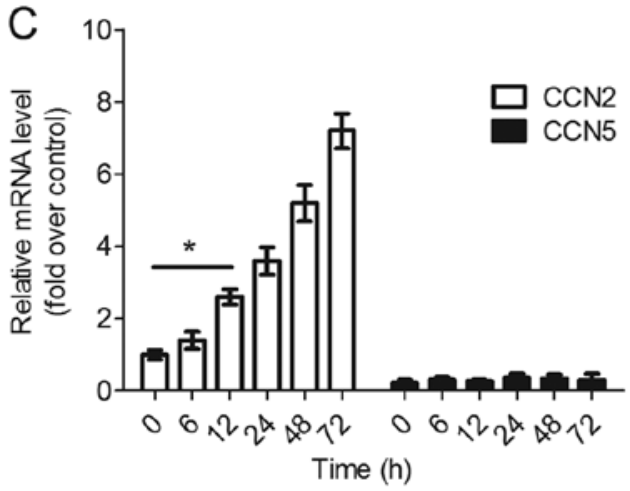

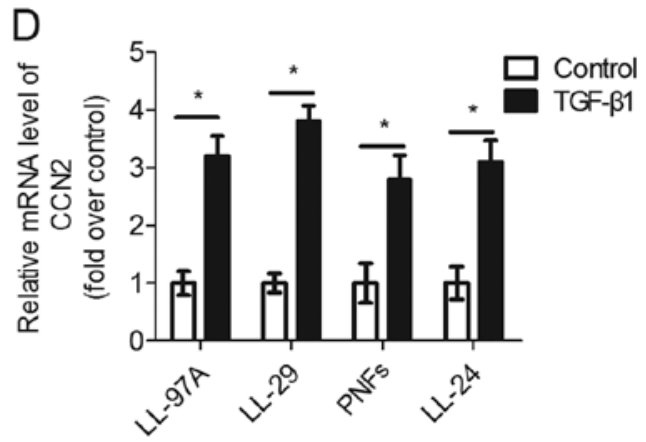

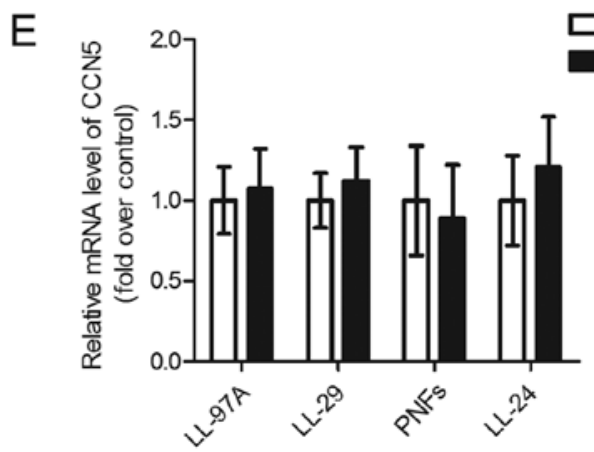

Figure 1. CCN2 and CCN5 expression patterns in pulmonary fibroblasts. (A) CCN2 and CCN5 expression patterns were detected by western blot analysis in primary lung fibroblasts derived from patients with idiopathic pulmonary fibrosis (PIFs), the human IPF fibrobastic cell lines, LL-97A and LL-29, and primary human normal lung fibroblasts (PNFs) and normal human fibroblastic cell line, LL-24. (B) PIFs was pre-incubated with TGF- $\beta 1$ (range, $0-8$ ng/ml) for 24 h. CCN2 and CCN5 expression was detected by qRT-PCR. (C) PIFs were pre-incubated with TGF- $\beta 1$ ( 2 ng/ml) for up to 72 h. CCN2 and CCN5 expression was detected byqRT-PCR. Cells were pre-treated with TGF- $\beta 1(2 \mathrm{ng} / \mathrm{ml})$ for $12 \mathrm{~h}$. (D) CCN2 and (E) CCN5 levels were determined by qRT-PCR. The mRNA expression levels was normalized based on $\beta$-actin levels. Relative mRNA expression levels were the ratio of the normalized mRNA expression levels in the treatment group to those in the control group. Data are expressed as the means \pm SEM of 3 independent experiments. P<0.05 was considered to indicate a statistically significant difference.

bleomycin. On day 14, the mice were sacrificed and samples of the lung tissue were used to determine the infection efficiency. Western blot analysis revealed that the administration of bleomycin markedly increased the expression of CCN2, but not that of CCN5 (Fig. 5A). Ad-CCN5 infection markedly increased the expression of CCN5 in the mice with bleomycininduced IPF $(\mathrm{P}<0.05)$, and markedly decreased the levels of CCN2 (Fig. 5A). To quantify the effects of treatment with Ad-CCN5 on collagen deposition in the lungs following the administration of bleomycin, we measured the hydroxyproline content in the lungs. The hydroxyproline content in the lungs was increased by approximately 4-fold in the mice with bleomycin-induced IPF compared with the normal control mice, consistent with the findings from previous studies (25)
(Fig. 5B). Pre-treatment with Ad-GFP did not affect the hydroxyproline content in the lungs (Fig. 5B). However, pre-treatment with Ad-CCN5 significantly suppressed the bleomycin-induced increase in hydroxyproline content in the lungs $(\mathrm{P}<0.05)$, indicating that $\mathrm{CCN} 5$ overexpression alleviated lung fibrosis in vivo (Fig. 5B). In light of the fact that TGF- $\beta$ is the main profibrotic factor in the majority of fibrotic diseases, we also evaluated the alterations in TGF- $\beta 1$ levels in BALF taken from the mice by ELISA. As expected, bleomycin administration significantly increased the TGF- $\beta 1$ levels (Fig. 5C). However, pre-treatment with Ad-CCN5 markeldy inhibited the bleomycin-induced increase in TGF- $\beta 1$ levels; however, pre-treatment with Ad-GFP had little effect on the secretion of TGF- $\beta 1$ (Fig. $5 \mathrm{C}$ ). 

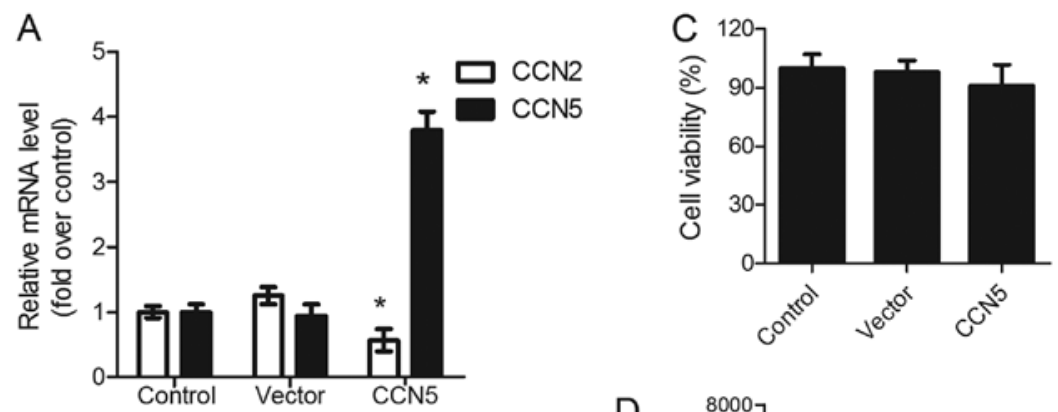

B
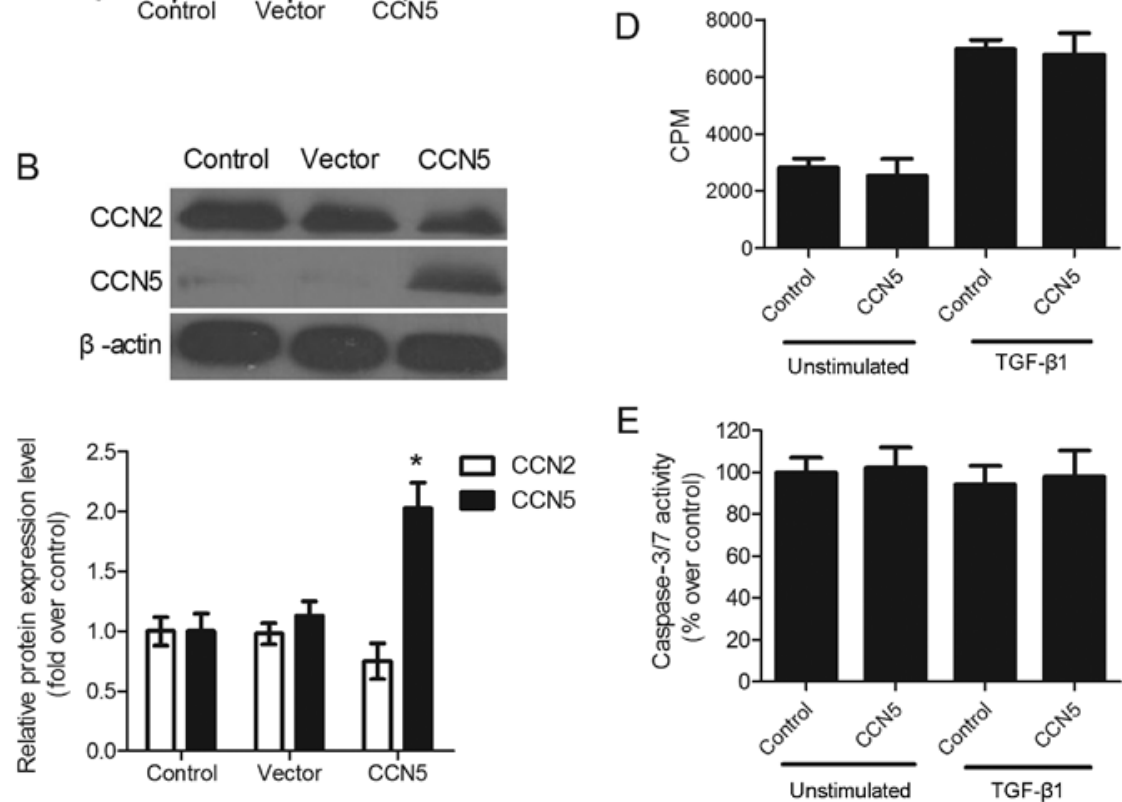

Figure 2. Effects of CCN5 overexpression on the viability, proliferation and apoptosis of pulmonary fibroblasts. Primary lung fibroblasts derived from patients with idiopathic pulmonary fibrosis (PIFs) were transfected with the pCEP4-Flag-CCN5 recombinant plasmid. The expression of CCN2 and CCN5 in the control group, vector group and pCEP4-Flag-CCN5-transfected group was determined by (A) qRT-PCR and (B) western blot analysis. (C) Cell viability was determined by a CellTiter-Blue Cell Viability assay kit. (D) Cell proliferation was determined by $\left[{ }^{3} \mathrm{H}\right]$-thymidine incorporation assay. Cells were left unstimulated or pre-incubated with TGF- $\beta 1$ for $24 \mathrm{~h}$. Data are expressed as the percentage incorporation of $\left[{ }^{3} \mathrm{H}\right]$-thymidine compared with the control group. CPM, counts per minute. (E) Cell apoptosis was determined by measuring the activity of caspase-3/7. Cells were left unstimulated or pre-incubated with TGF- $\beta 1$ for $24 \mathrm{~h}$. Data are expressed as means \pm SEM of 3 independent experiments. $\mathrm{P}<0.05$ was considered to indicate a statistically significant difference.
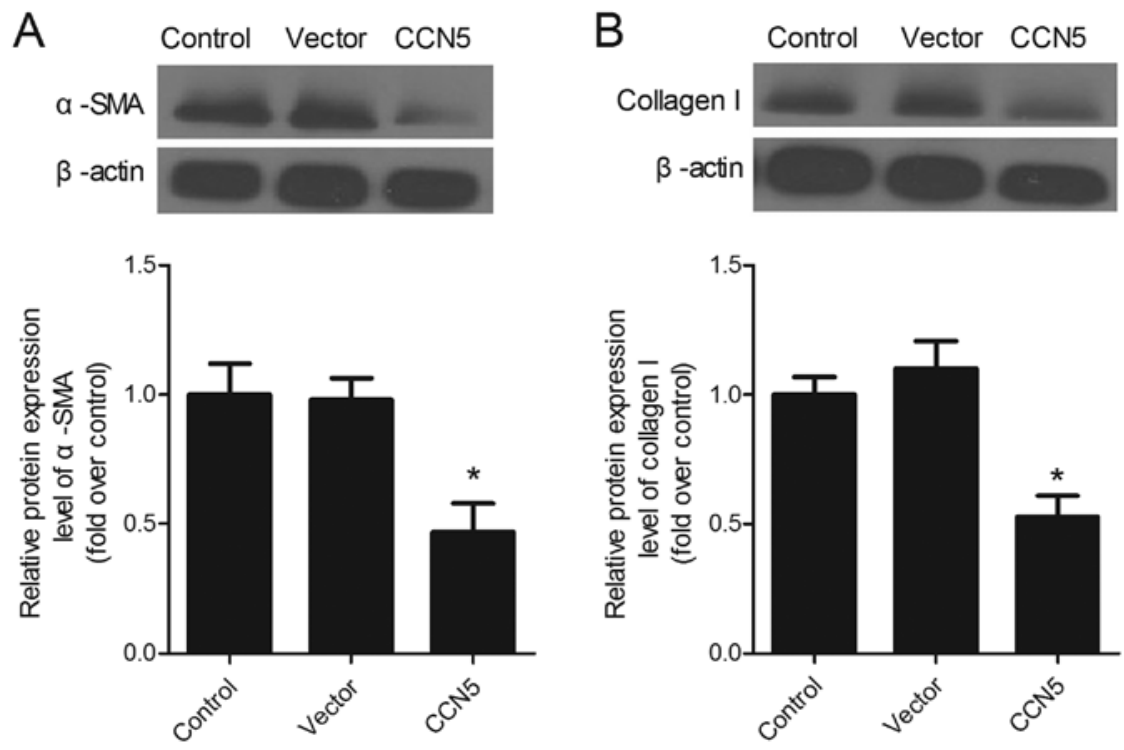

Figure 3. Effect of CCN5 overexpression on the expression of $\alpha$-smooth muscle actin ( $\alpha$-SMA) and collagen type I. Primary lung fibroblasts derived from patients with idiopathic pulmonary fibrosis (PIFs) were transfected with the pCEP4-Flag-CCN5 recombinant plasmid. Following stimulation with $2 \mathrm{ng} / \mathrm{ml}$ TGF- $\beta 1$ for $24 \mathrm{~h}$, the cells were examined for (A) $\alpha$-SMA and (B) collagen I expression assay by western blot analysis. Data are expressed as the means \pm SEM of 3 independent experiments. $\mathrm{P}<0.05$ was considered to indicate a statistically significant difference. 

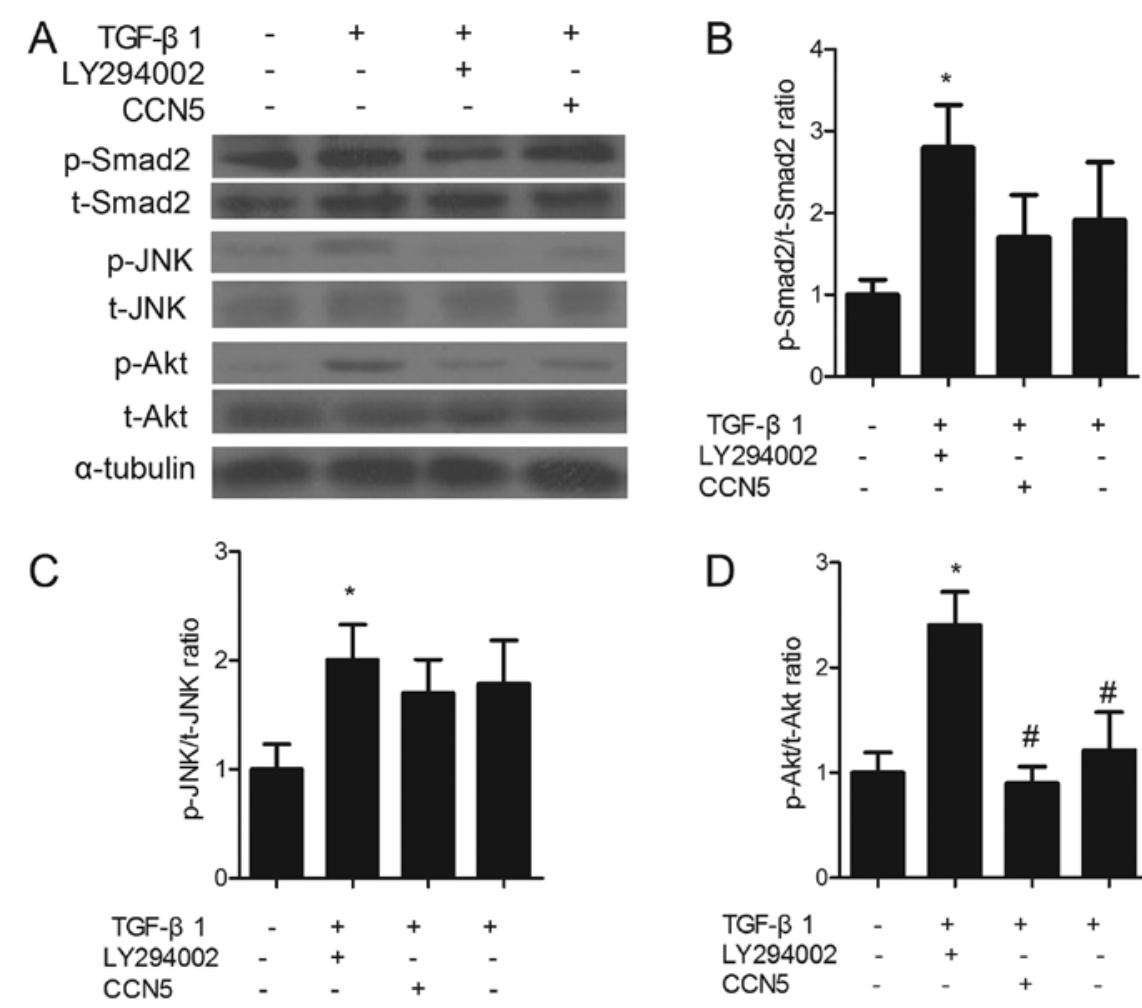

Figure 4. (CCN5 overexpression attenuates the phosphorylation of Akt. Primary lung fibroblasts derived from patients with idiopathic pulmonary fibrosis (PIFs) were either left untreated, stimulated with TGF- $\beta 1$, pre-treated with PI3K inhibitor ,LY294002, or transfected with pCEP4-Flag-CCN5 prior to TGF- $\beta 1$ stimulation. (A) Western blot analysis was used to determine the levels of phosphorylated Smad2 (p-Smad2) and total Smad2 (t-Smad2), JNK and Akt. The intensity of the bands probed with (B) anti-p-Smad2, (C) anti-p-JNK and (D) anti-p-Akt antibodies was quantified and normalized to the corresponding total protein. Data are expressed as the means \pm SEM of 3 independent experiments. ${ }^{~} \mathrm{P}<0.05$ vs. the control group and ${ }^{\#} \mathrm{P}<0.05$ vs. the TGF- $\beta 1$ treated group.
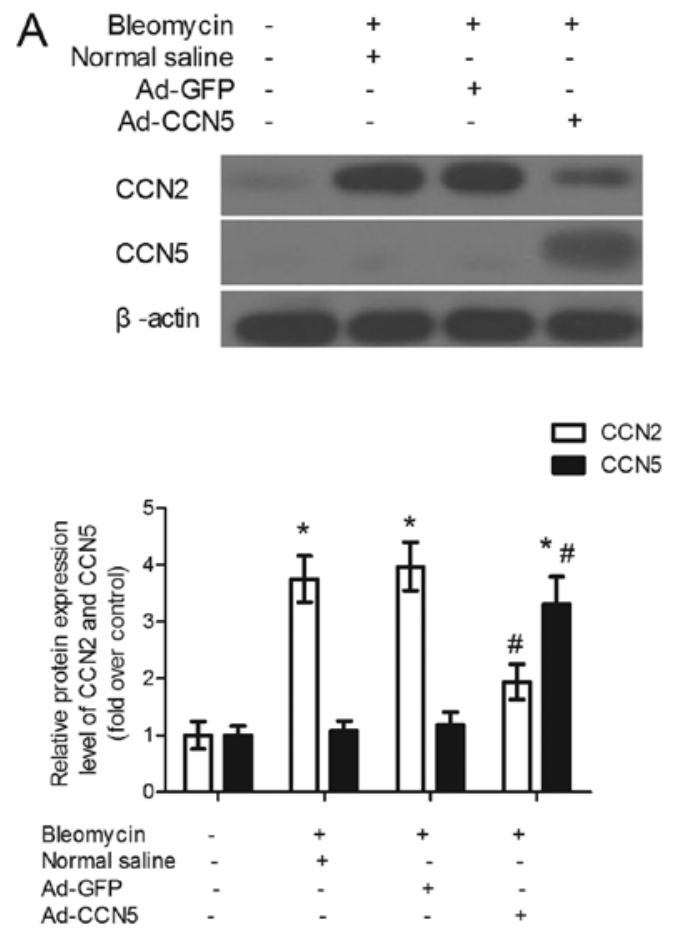
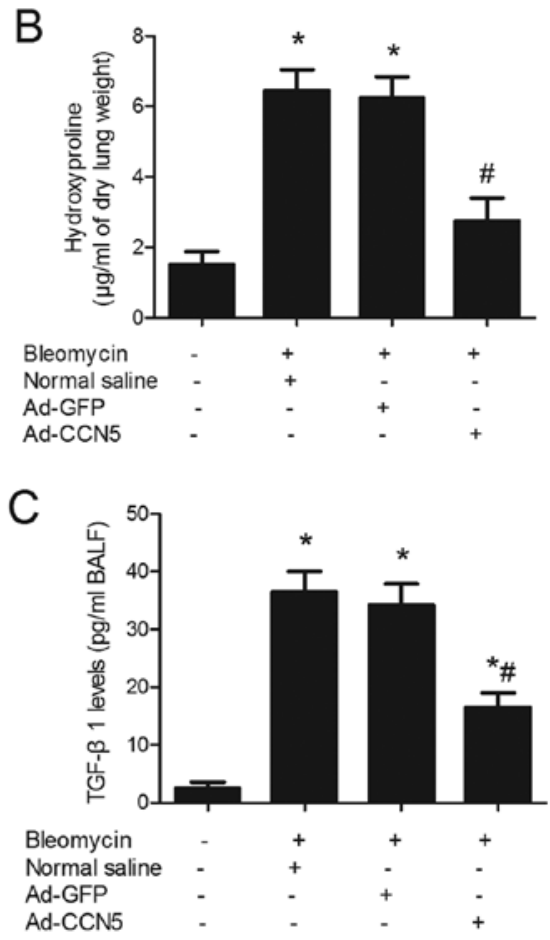

Figure 5. Effects CCN5 overexpression on fibrotic response in lungs of mice with bleomycin-induced lung fibrosis. Mice were either left untreated, pre-treated with normal saline, pre-treated with Ad-GFP or pre-treated with Ad-CCN5 prior to the administration of bleomycin. (A) Mice were sacrificed on day 14 and samples of the lung tissue were used for protein extraction. CCN2 and CCN5 expression levels were determined by western blot analysis. (B) Hydroxyproline content at 14 days in the lungs of mice with bleomycin-induced lung fibrosis was determined using a hydroxyproline assay. (C) Bronchoalveolar lavage fluid (BALF) was collected on day 14, and levels of TGF- $\beta 1$ in the BALF were measured with an ELISA kit using a standardized procedure. Data are expressed as the means \pm SEM. ${ }^{*} \mathrm{P}<0.05$ vs. the normal untreated group and ${ }^{*} \mathrm{P}<0.05$ vs. the group pre-treated with normal saline and administered bleomycin ( $\mathrm{n}=10$ ). 


\section{Discussion}

Although CCN5 has been suggested to play an anti-fibrotic role in some fibrotic diseases, the role of CCN5 in IPF remains unknown. In the present study, we demonstrate that CCN5 overexpression inhibits the fibrotic phenotype in vitro and in vivo.

IPF is a specific form of chronic fibrotic interstitial pneumonia with a poor prognosis. The concept of IPF pathogenesis has progressed from chronic inflammation to aberrant wounding healing and, even more recently, to the current paradigms of a multifactorial and heterogeneous disease process (30). However, the molecular pathogenetic mechanisms of IPF remain unclear and there are few effective therapies. Therefore, the identification of novel anti-fibrotic targets for the treatement of IPF is urgently required. Apigenin (4',5,7,-trihydroxyflavone), a type of flavonoid, has been shown to have the ability to inhibit the TGF- $\beta 1$-induced fibroblast-tomyofibroblast transition in human lung fibroblasts (31). Previous studies have also emphasized the important role of CCN2, a downstream mediator of TGF- $\beta$ signaling, in the pathogenesis of IPF (16). In this study, we demonstrated that CCN2 was highly expressed in lung fibroblasts isolated from patients with IPF (PIFs) and cell lines derived from IPF patients, and that its expression increased by TGF- $\beta 1$ stimulation. These data are consistent with those of previous reports $(15,16)$. We also determined that the expression of CCN5 was low in PIFs, normal lung fibroblasts and cell lines. TGF- $\beta 1$ stimulation markedly increased the expression of $\mathrm{CCN} 2$, but not that of CCN5. This indicated that CCN5 was not regulated by TGF- $\beta$ signaling. TGF- $\beta$-Smad is a vital signaling pathway associated with CCN2 expression and fibrosis. In this pathway, TGF- $\beta$ stimulation induces the phosphorylation of Smad2 and Smad3 and the formation of complexes with Smad4. These complexes are then translocated into the nucleus and activate the transcription of target genes.

Previous studies have revealed that the upregulation of collagen and TGF- $\beta$ are markedly exacerbated in CCN2 transgenic mice, but are inhibited in CCN5 transgenic mice (20). In light of the opposing roles of CCN2 and CCN5 in cardiac fibrosis (20), we hypothesized that CCN5 may inhibit the profibrotic effects of TGF- $\beta 1$. In accordance with our hypothesis, the transfection of lung fibroblasts with a recombined plasmid expressing CCN5 notably decreased the expression of $\alpha$-SMA and collagen induced by TGF- $\beta 1$. We also demonstrated that the mRNA levels of CCN2 were suppressed by CCN5 overexpression, although a variation in $\mathrm{CCN} 2$ protein levels was not obvious, indicating that CCN5 may be involved in the signaling pathway associated with $\mathrm{CCN} 2$ transcription. However, the effects of CCN5 silencing on lung fibroblasts were not investigated in the present study as the spontaneous expression level of CCN5 was weak in the lung fibroblasts even after stimulation with TGF- $\beta 1$. In a previous study, it was demonstrated that the silencing of CCN5 significantly upregulated several components of the TGF- $\beta$ signaling pathway, including CCN2, fibronectin, Smad3 and the TGF- $\beta$ type I and II receptors, but downregulated the expression of Smad6, an inhibitory component of this pathway (21). This suggested that CCN5 antagonizes TGF- $\beta$ signaling. The depletion of CCN5 in the breast cancer cell line, MCF-7, also culminated in an epithelial mesenchymal transition (EMT) (21). In another study, the level of Smad7, another inhibitory component of the TGF- $\beta$-Smad pathway, remained elevated in CCN5 transgenic mice, but was significantly reduced in CCN2 transgenic mice (20). None of these data contradicted our findings. Furthermore, using a chromatin immunoprecipitation assay, CCN5 has also been suggested to prevent the transcription of TGF- $\beta$ type II receptor by recruiting histone deacetylase to the TGF- $\beta$ type II receptor promoter, thus leading to its deacetylation (20). However, the phosphorylation of Smad2 and the Smad4 protein levels was slightly decreased in CCN5 transgenic mice (20), suggesting that other components are also involved in the TGF- $\beta$-Smad pathway.

Apart from the Smad-dependent pathway, Smadindependent pathways, such as PI3K/Akt have also been suggested to be involved in TGF- $\beta$ signaling $(32,33)$. In the present study, we demonstrated that CCN5 overexpression significantly abrogated the phosphorylation of Akt induced by TGF- $\beta 1$. Furthermore, CCN5 overexpression markedly reduced fibroblast-to-myoblast differentiation; however, it had little affect on the viability, apoptosis and proliferation of lung fibroblasts (Figs. 2 and 3). These data was partly in agreement with the finding that the inhibition of PI3K prevents the proliferation and differentiation of human lung fibroblasts into myofibroblasts (29). CCN5 has also been shown to possess the ability to suppress proliferation in certain types of cancer $(34,35)$. This discrepancy in results may be due to the fact that CCN5 has a weaker inhibitory effect than the PI3K inhibitor, LY294002, or to the different cell types used.

We then investigated the effects of CCN5 in mcie with bleomycin-induced IPF using recombinant adenoviral vectors expressing CCN5. CCN5 overexpression in the lungs abrogated the bleomycin-induced upregulation of CCN2 and collagen in the tissue. These in vivo data are in accordance with our findings on lung fibroblasts in vitro. We also revealed that the TGF- $\beta 1$ level in BALF was reduced. However, the mechanisms these effects remain unclear.

In conclusion, our results demonstrate that CCN5 exerts an inhibitory effect on the fibrotic phenotypes in lung fibroblasts derived from patients with IPF and in vivo models of lung fibrosis, suggesting that CCN5 is a promising target for the treatment of IPF. Nevertheless, the precise molecular mechanisms underlying the anti-fibrotic role of CCN5 in IPF have not been fully elucidated and require further investigation.

\section{Acknowledgements}

This study was supported by a grant from the Technology Plan Projects of Shaanxi province (no. 2011k14-10-01). The funders had no role in the study design, data collection and analysis, decision to publish, or preparation of the manuscript.

\section{References}

1. Huang LS, Berdyshev E, Mathew B, et al: Targeting sphingosine kinase 1 attenuates bleomycin-induced pulmonary fibrosis. FASEB J 27: 1749-1760, 2013.

2. Scotton CJ and Chambers RC: Molecular targets in pulmonary fibrosis: the myofibroblast in focus. Chest 132: 1311-1321, 2007.

3. Singh SR and Hall IP: Airway myofibroblasts and their relationship with airway myocytes and fibroblasts. Proc Am Thorac Soc 5: 127-132, 2008. 
4. Phan SH: The myofibroblast in pulmonary fibrosis. Chest 122 : 286S-289S, 2002.

5. Kono M, Nakamura Y, Suda T, et al: Plasma CCN2 (connective tissue growth factor; CTGF) is a potential biomarker in idiopathic pulmonary fibrosis (IPF). Clin Chim Acta 412: 2211-2215, 2011.

6. Fernandez IE and Eickelberg O: The impact of TGF- $\beta$ on lung fibrosis: from targeting to biomarkers. Proc Am Thorac Soc 9: 111-116, 2012.

7. Fine A and Goldstein RH: The effect of transforming growth factor-beta on cell proliferation and collagen formation by lung fibroblasts. J Biol Chem 262: 3897-3902, 1987.

8. Leask A and Abraham DJ: All in the CCN family: essential matricellular signaling modulators emerge from the bunker J Cell Sci 119: 4803-4810, 2006.

9. Inadera H, Hashimoto S, Dong HY, et al: WISP-2 as a novel estrogen-responsive gene in human breast cancer cells. Biochem Biophys Res Commun 275: 108-114, 2000.

10. Moussad EE and Brigstock DR: Connective tissue growth factor: what's in a name? Mol Genet Metab 71: 276-292, 2000.

11. Ponticos M, Holmes AM, Shi-wen X, et al: Pivotal role of connective tissue growth factor in lung fibrosis: MAPKdependent transcriptional activation of type I collagen. Arthritis Rheum 60: 2142-2155, 2009.

12. Brigstock DR: Connective tissue growth factor (CCN2, CTGF) and organ fibrosis: lessons from transgenic animals. J Cell Commun Signal 4: 1-4, 2010.

13. Sonnylal S, Shi-Wen X, Leoni P, et al: Selective expression of connective tissue growth factor in fibroblasts in vivo promotes systemic tissue fibrosis. Arthritis Rheum 62: 1523-1532, 2010.

14. Liu S, Shi-wen X, Abraham DJ and Leask A: CCN2 is required for bleomycin-induced skin fibrosis in mice. Arthritis Rheum 63 : 239-246, 2011

15. Chambers RC, Leoni P, Blanc-Brude OP, Wembridge DE and Laurent GJ: Thrombin is a potent inducer of connective tissue growth factor production via proteolytic activation of protease-activated receptor-1. J Biol Chem 275: 35584-35591, 2000.

16. Bonniaud P, Martin G, Margetts PJ, et al: Connective tissue growth factor is crucial to inducing a profibrotic environment in 'fibrosis-resistant' BALB/c mouse lungs. Am J Respir Cell Mol Biol 31: 510-516, 2004

17. Fritah A, Redeuilh G and Sabbah M: Molecular cloning and characterization of the human WISP-2/CCN5 gene promoter reveal its upregulation by oestrogens. J Endocrinol 191: 613-624, 2006.

18. Brigstock DR: The connective tissue growth factor/cysteine-rich 61/nephroblastoma overexpressed (CCN) family. Endocr Rev 20: 189-206, 1999.

19. Leask A: Yin and Yang Part Deux: CCN5 inhibits the pro-fibrotic effects of CCN2. J Cell Commun Signal 4: 155-156, 2010.

20. Yoon PO, Lee MA, Cha H, et al: The opposing effects of CCN2 and CCN5 on the development of cardiac hypertrophy and fibrosis. J Mol Cell Cardiol 49: 294-303, 2010.

21. Sabbah M, Prunier C, Ferrand N, et al: CCN5, a novel transcriptional repressor of the transforming growth factor $\beta$ signaling pathway. Mol Cell Biol 31: 1459-1469, 2011.
22. Ricci A, Cherubini E, Ulivieri A, et al: Homeodomain-interacting protein kinase 2 in human idiopathic pulmonary fibrosis. J Cell Physiol 228: 235-241, 2013.

23. Livak KJ and Schmittgen TD: Analysis of relative gene expression data using real-time quantitative PCR and the 2(-Delta Delta C(T)) Method. Methods 25: 402-408, 2001.

24. Fritah A, Saucier C, De Wever O, et al: Role of WISP-2/CCN5 in the maintenance of a differentiated and noninvasive phenotype in human breast cancer cells. Mol Cell Biol 28: 1114-1123, 2008

25. Tsuburai T, Suzuki M, Nagashima Y, et al: Adenovirus-mediated transfer and overexpression of heme oxygenase $1 \mathrm{cDNA}$ in lung prevents bleomycin-induced pulmonary fibrosis via a Fas-Fas ligand-independent pathway. Hum Gene Ther 13: 1945-1960, 2002.

26. Woessner JF, Jr.: The determination of hydroxyproline in tissue and protein samples containing small proportions of this imino acid. Arch Biochem Biophys 93: 440-447, 1961.

27. Milam JE, Keshamouni VG, Phan SH, et al: PPAR-gamma agonists inhibit profibrotic phenotypes in human lung fibroblasts and bleomycin-induced pulmonary fibrosis. Am J Physiol Lung Cell Mol Physiol 294: L891-901, 2008.

28. Kolb M, Margetts PJ, Galt T, et al: Transient transgene expression of decorin in the lung reduces the fibrotic response to bleomycin. Am J Respir Crit Care Med 163: 770-777, 2001

29. Conte E, Fruciano M, Fagone E, et al: Inhibition of PI3K prevents the proliferation and differentiation of human lung fibroblasts into myofibroblasts: the role of class I P110 isoforms. PLoS One 6: e24663, 2011

30. Park S and Lee EJ: Recent advances in idiopathic pulmonary fibrosis. Tuberc Respir Dis (Seoul) 74: 1-6, 2013.

31. Wójcik KA, Skoda M, Koczurkiewicz P, Sanak M, Czyż J and Michalik M: Apigenin inhibits TGF- $\beta 1$ induced fibroblast-tomyofibroblast transition in human lung fibroblast populations. Pharmacol Rep 65: 164-172, 2013.

32. Wilkes MC, Mitchell H,Penheiter SG, et al: Transforming growth factor-beta activation of phosphatidylinositol 3-kinase is independent of Smad2 and Smad3 and regulates fibroblast responses via p21-activated kinase-2. Cancer Res 65: 10431-10440, 2005.

33. Martin MM, Buckenberger JA, Jiang J, et al: TGF-beta1 stimulates human AT1 receptor expression in lung fibroblasts by cross talk between the Smad, p38 MAPK, JNK, and PI3K signaling pathways. Am J Physiol Lung Cell Mol Physiol 293 L790-L799, 2007.

34. Mason HR, Lake AC, Wubben JE, Nowak RA and Castellot JJ Jr: The growth arrest-specific gene CCN5 is deficient in human leiomyomas and inhibits the proliferation and motility of cultured human uterine smooth muscle cells. Mol Hum Reprod 10: 181-187, 2004.

35. Pennica D, Swanson TA, Welsh JW, et al: WISP genes are members of the connective tissue growth factor family that are up-regulated in wnt-1-transformed cells and aberrantly expressed in human colon tumors. Proc Natl Acad Sci USA 95: $14717-14722,1998$ 